\title{
A Delayed Diagnosis of an Isolated Trapezoidal Fracture due to Direct Trauma: A Case Report
}

\author{
Rikito Tatsuno $^{1}$, Tetsuo Hagino ${ }^{1 *}$, Satoshi Ochiai ${ }^{1}$, Shinya Senga ${ }^{1}$, Takashi Yamashita ${ }^{1}$, Masanori \\ Wako $^{2}$, Naofumi Taniguchi ${ }^{2}$, Takashi Ando ${ }^{2}$, Hirotaka Haro ${ }^{2}$ \\ ${ }^{I}$ Department of Orthopaedic Surgery, National Hospital Organization Kofu National Hospital, Tenjin-cho, Kofu, \\ Yamanashi, Japan
}

${ }^{2}$ Department of Orthopaedic Surgery, Faculty of Medicine, University of Yamanashi, Yamanashi, Japan

*Corresponding Author: Tetsuo Hagino, Department of Orthopaedic Surgery, National Hospital Organization Kofu National Hospital, Tenjin-cho, Kofu, Yamanashi, Japan, Email: tmhagino@amber.plala.or.jp

\begin{abstract}
An isolated trapezoid fracture is very rare, because of the anatomical shape and position of the trapezoid bone and the strong ligament attachments with adjacent bones. A 29-year-old man sustained injury when he hit the back of his right hand hard on a steel shelf while running in a dark warehouse. Two weeks after injury, he presented at our hospital with mild swelling on the dorsal radius of the right wrist and tenderness at the snuff box. Although there were no abnormalities on plain radiographs, MRI and CT demonstrated isolated trapezoid fracture. Conservative treatment using cast immobilization was performed. At 12 months after injury, the clinical course was uneventful with no impairment of ADL. An isolated trapezoid fracture with no displacement appears normal on plain radiographs. A CT scan or MRI is essential for a definitive diagnosis.
\end{abstract}

Keywords: Trapezoid, Isolated Fracture, Delayed Diagnosis

\section{INTRODUCTION}

Carpal fractures are frequently encountered in routine clinical practice. Discounting the metacarpals and phalanges, carpal bone fractures account for approximately $8 \%$ of all hand fractures [1].Among carpal bone fractures, scaphoid fracture is the most common, constituting $68-79 \%[2,3]$. On the other hand, trapezoid bone fracture is rare. In this report, we describe a rare case of isolated trapezoid fracture encountered clinically.

\section{CASE RePort}

A 29-year-old right-hand dominant male visited a local orthopedic clinic because of pain at the dorsum of the right hand. One day before the visit, he hit the back of his right hand hard on a steel shelf while running in a dark warehouse. No abnormality was observed on a plain radiograph, and he was followed without treatment. Since the pain at the dorsum of the right hand did not improve, he was referred to our department 2 weeks after injury.
At presentation, physical examination was remarkable except for soft tissue swelling at the dorsal hand and point tenderness at the "snuff box". Plain radiographs of the patient's wrist showed no abnormalities (Figure1) Because of tenderness at the snuff box, scaphoid fracture was suspected, and MRI was performed. MRI showed no abnormalities in the scaphoid bone, but revealed low signal intensity on T1-weight image and high signal intensity on T2-weighted fat-suppressed image in the trapezoid bone (Figure2). Sagittal section of a CT scan showed a fracture line from the distal to proximal side of the trapezoid bone (Figure3). Since there were no other fractures and dislocations in the right wrist, isolated trapezoid fracture was diagnosed and was treated with cast immobilization. Three weeks after presentation, the cast was replaced by a plaster splint. Immobilization was terminated after six weeks. At the follow-up 12 months after injury, there was no tenderness or swelling, and the clinical course was uneventful without impairment of ADL. 


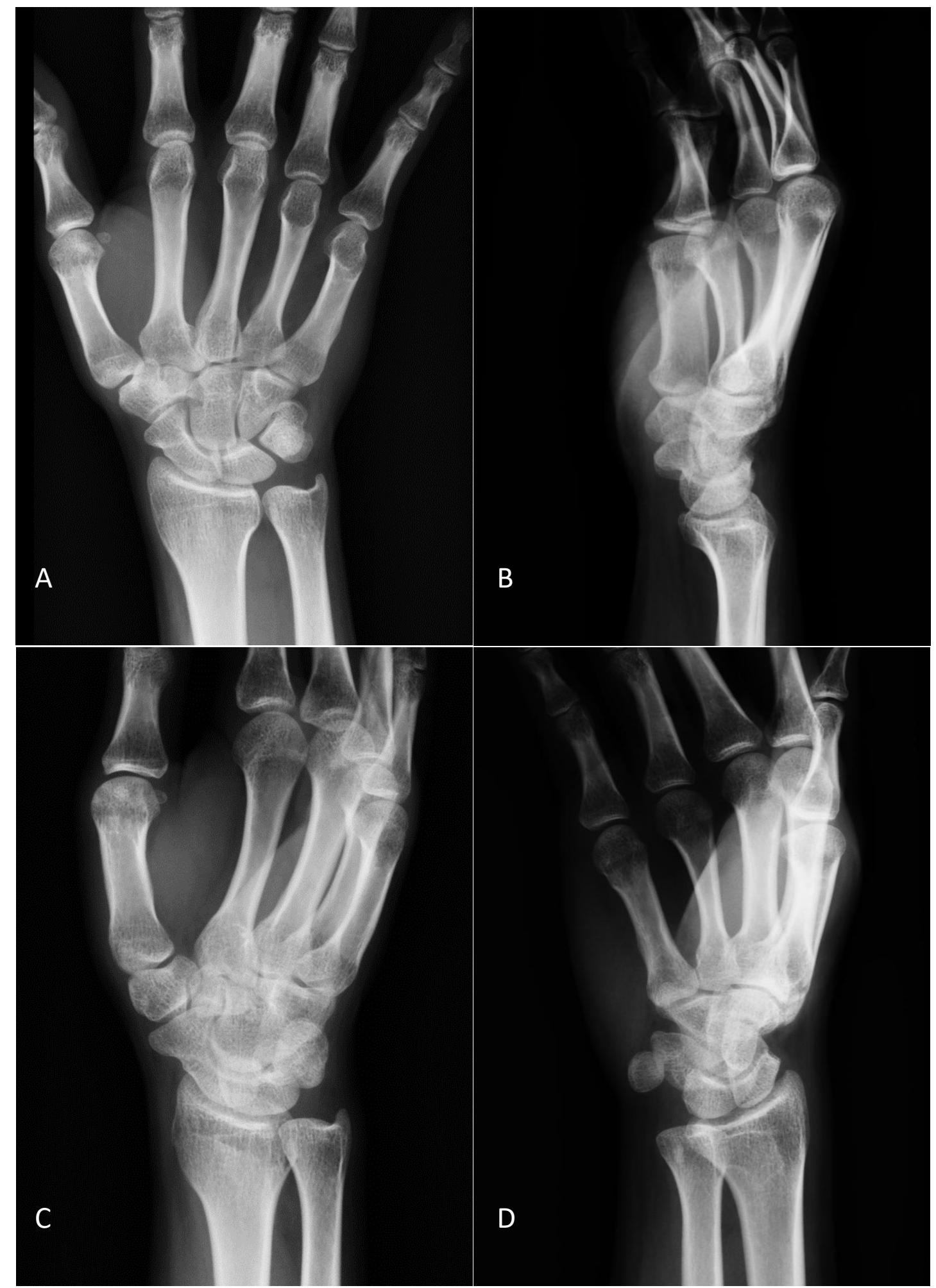

Figure1: Anteroposterior (A), lateral $(B)$ and oblique(C, D) radiographs show apparently normal anatomy without fracture or dislocation. 


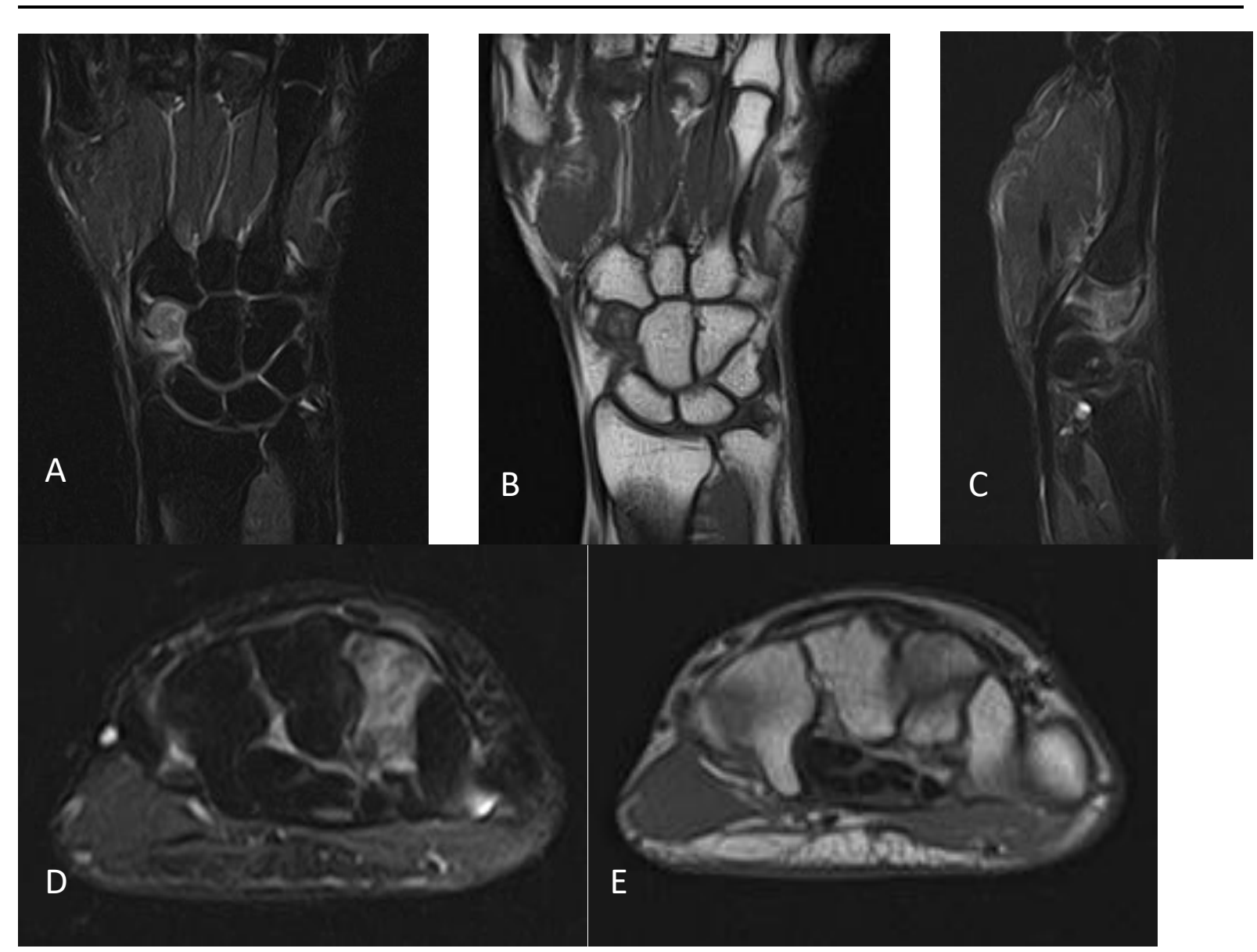

Figure2: MRI revealed abnormal signal intensities. (A) Coronal T lipid-suppressed image, (B) coronal T1weighted image, $(D, E)$ axial T1- and T2-weighted fat-suppressed images.

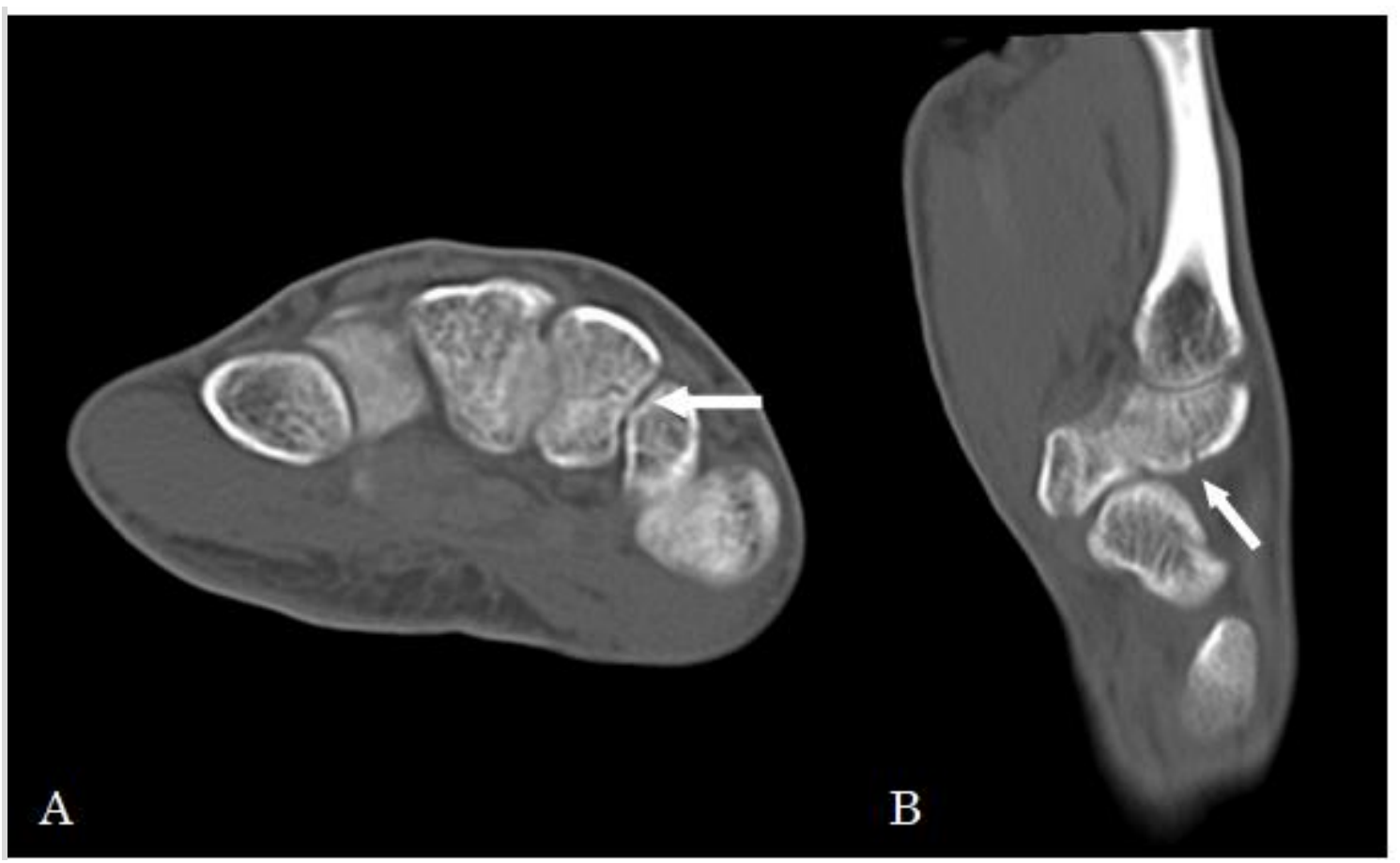

Figure3: Axial (A) and sagittal (B) views of a computed tomography scan of the wrist demonstrating trapezoid fracture. While arrow indicates the fracture line

\section{DISCUSSION}

The trapezoid bone is the smallest bone in the distal row of the carpal bones, and is an inherently stable keystone-shaped bone with ARC Journal of Clinical Case Reports strong ligament us attachments. The bone is set within the second metacarpal base, and bound by strong ligaments to the trapezium, capitate, and scaphoid. Because of the ligament us 
stability and relatively protected position within the interlocking carpal geometry, the trapezoid is less commonly injured than any other carpal bone $[4,5]$.

Trapezoid fractures are uncommon injuries, representing 0.2-0.4\% of all carpal bone fractures[6, 7]. However, this incidence includes compound fractures, and isolated trapezoid injuries are even rarer. Blomquist et al. [8] investigated 64 cases of trapezoid fractures. In their series, $95 \%$ of the fractures were combined with other wrist injuries, and the three cases of isolated trapezoid fractures represented only $4.7 \%$ of the total number of trapezoid fractures.

Trapezoid fractures are sustained during punching, or when the hand impacts a hard surface when falling during sport activities or driving a motorcycle. The mechanism of trapezoid fracture is an axially transmitted force or a bending force acting over the index metacarpal $[4,5,9]$, or direct trauma to the metacarpal base[10]. In the present case, the fracture was probably due to direct trauma to the trapezoid bone.

Standard anteroposterior, lateral, and oblique radiographs should be obtained initially to visualize the osseous structures of the wrist. An isolated trapezoid fracture is easily missed on plain radiographs, especially with no displaced fractures. Welling et al. [11] reported that 30\% of carpal fractures were overlooked on plain radiographs, and were successfully diagnosed by CT. Trapezoid fracture is very difficult to diagnose with X-ray alone, due to the overlapping bones and the small size of bone fragments. CT and MRI are very useful for a definitive diagnosis [5, 12, 13]. Furthermore, technetium 99m methylene diphosphonate (MDP) bone scan has been reported to be useful $[4,12]$.

For trapezoid fractures on the sagittal plane with little displacement as in the present case, the diagnosis may be delayed. Hence, MRI and CT studies are necessary. While early CT or MRI examination leads to a prompt diagnosis, performing only X-ray may delay the diagnosis by 2 to 4 weeks $[4,5]$.

There are few cases that require surgery. Most of the cases achieved favorable outcome with cast immobilization for 3 to 6 weeks followed by wearing a device [4-6, 9, 12, and 14]. Jeong et al. [4] and Al-Sadek et al. [13] concluded that treatment of no displaced or minimally displaced $(<2 \mathrm{~mm})$ isolated trapezoid fractures with cast immobilization appears to result in good union and functional outcomes. The present case showed no displacement, and external fixation for 6 weeks achieved good outcome without pain or impairment of ADL.

\section{CONCLUSION}

We encountered a rare case of isolated trapezoid fracture. This fracture is difficult to diagnose by plain radiography alone. When trapezoid fracture is suspected, MRI and CT should be performed early. Conservative treatment of trapezoid fracture without displacement achieves favorable outcome.

\section{REFERENCES}

[1] Van Onselen, E.B.H., et al., Prevalence and Distribution of Hand Fractures. JHand Surg Br, 2003. 28(5): p. 491-495.

[2] Smith, D.K., et al., The effects of simulated unstable scaphoid fractures on carpal motion. J Hand Surg Am, 1989. 14(2 Pt 1): p. 283-91.

[3] Papp, S., Carpal bone fractures. Orthop Clin North Am, 2007. 38(2): p. 251-60, vii.

[4] Jeong, G.K., D. Kram, and B. Lester, Isolated fracture of the trapezoid. Am J Orthop (Belle Mead NJ), 2001. 30(3): p. 228-30.

[5] Nijs, S., T. Mulier, and P. Broos, Occult fracture of the trapezoid bone: a report on two cases. Acta Orthop Belg, 2004. 70(2): p. 177-9.

[6] Sadowski, R.M. and R.D. Montilla, Rare isolated trapezoid fracture: a case report. Hand (New York, N.Y.), 2008. 3(4): p. 372-374.

[7] Gupta, P.K., et al., Fracture of the trapezoid bone: An unusual fracture. J Orthop Allied Sci, 2015. 3(1): p. 28.

[8] Blomquist, G.A., T.R. Hunt III, and R.R. Lopez-Ben, Isolated fractures of the trapezoid as a sports injury. Skeletal Radiol, 2013. 42(5): p. 735-739.

[9] Miyawaki, T., et al., Trapezoid bones fracture. Ann Plast Surg, 2000. 44(4): p.444-6.

[10] Pruzansky, M. and L. Arnold, Delayed union of fractures of the trapezoid and body of the hamate. Orthop Rev, 1987. 16(9): p. 624-8.

[11] Welling, R.D., et al., MDCT and radiography of wrist fractures: radiographic sensitivity and 
fracture patterns. AJR Am J Roentgenol, 2008. 190(1): p. 10-6.

[12] Nagumo, A., et al., An occult fracture of the trapezoid bone. A case report. J Bone Joint Surg Am, 2002. 84-a (6): p. 1025-7.
[13] Al-Sadek, T., et al., A rare case of trapezoid fractures. J Orthop Ther: JORT-149, 2017.

[14] Gruson, K.I., K.M. Kaplan, and N. Paksima, Isolated Trapezoid Fractures. Bull NYU Hosp Jt Dis, 2008. 66(1): p. 57-60.

Citation: Rikito Tatsuno, Tetsuo Hagino et.al. A Delayed Diagnosis of an Isolated Trapezoidal Fracture due to Direct Trauma: A Case Report. ARC Journal of Clinical Case Reports. 2020; 6(1): 1-5. doi:dx.doi.org/ 10.20431/2455-9806.0601001.

Copyright: (c) 2020 Authors. This is an open-access article distributed under the terms of the Creative Commons Attribution License, which permits unrestricted use, distribution, and reproduction in any medium, provided the original author and source are credited. 